\title{
A TRACIAL NULLSTELLENSATZ
}

\author{
IGOR KLEP ${ }^{1}$ AND ŠPELA ŠPENKO ${ }^{2}$
}

\begin{abstract}
The main result of this note is a tracial Nullstellensatz for free noncommutative polynomials evaluated at tuples of matrices of all sizes: Suppose $f_{1}, \ldots, f_{r}, f$ are free polynomials, and $\operatorname{tr}(f)$ vanishes whenever all $\operatorname{tr}\left(f_{j}\right)$ vanish. Then either 1 or $f$ is a linear combination of the $f_{j}$ modulo sums of commutators.
\end{abstract}

\section{INTRODUCTION}

Hilbert's Nullstellensatz is a classical result in algebraic geometry. Over an algebraically closed field it characterizes polynomials vanishing on the zero set of a set of polynomials. Due to its importance it has been generalized and extended in many different directions, including to free algebras. For instance, Amitsur's Nullstellensatz [Ami57] describes free noncommutative polynomials vanishing on the zero set of a given finite set of free polynomials in a full matrix algebra. In another direction, the Nullstellensatz of Bergman [HM04] studies a weaker, directional notion of vanishing but in a dimension-independent context (see [CHMN13] for recent generalizations) allowing for a stronger conclusion. Namely, unlike in Hilbert's and Amitsur's Nullstellensatz, no powers are needed in the obtained algebraic certificate. We also refer the reader to [BK11] for a survey of free Nullstellensätze.

In this short article we focus on vanishing trace of free noncommutative polynomials. The relationship between sums of commutators and vanishing trace of a free polynomial is discussed e.g. in [CGM09, KS08, BK11]. Our main result, Theorem 3.1, characterizes free polynomials $f$ whose trace vanishes whenever the traces of polynomials $f_{1}, \ldots, f_{r}$ vanish. It is presented in Section 3, after preliminaries in Section 2. The main ingredients in the proof of our main result are effective degree bounds on Hilbert's Nullstellensatz due to Kollár [Kol88] (see also Sombra [Som99] and Jelonek [Jel05]), as well as the theory of polynomial identities [Row80, Pro76]. Finally, in Section 4 we solve a tracial moment problem by dualizing the statement of Theorem 3.1 .

Date: March 22, 2021.

2010 Mathematics Subject Classification. Primary 16R30, 47A57; Secondary 16S50, 15 A24.

Key words and phrases. free algebra, trace, Nullstellensatz, polynomial identity, degree bounds.

${ }^{1}$ Supported by the Faculty Research Development Fund (FRDF) of The University of Auckland (project no. 3701119). Partially supported by the Slovenian Research Agency grant P1-0222.

${ }^{2}$ Supported by the Slovenian Research Agency and in part by the Slovene Human Resources Development and Scholarship Fund. 


\section{Preliminaries}

2.1. Notation. Let $\mathbb{F}$ be a field of characteristic 0 and let $M(\mathbb{F})$ stand for $\bigcup_{n} M_{n}(\mathbb{F})$. We denote the free associative algebra in the variables $x_{1}, \ldots, x_{g}$ by $\mathbb{F}\langle X\rangle$. The free noncommutative polynomials in $\mathbb{F}\langle X\rangle$ of degree at most $d$ are denoted by $\mathbb{F}\langle X\rangle_{d}$, while $\mathbb{F}\langle X\rangle_{d}^{\prime}$ is the vector subspace of $\mathbb{F}\langle X\rangle_{d}$ consisting of all elements with zero constant term. We denote by $\langle X\rangle$ the monoid generated by $x_{1}, \ldots, x_{g}$, and by $\langle X\rangle_{d}$ words in $\langle X\rangle$ of degree at most $d$.

We say that polynomials $f, h \in \mathbb{F}\langle X\rangle$ are cyclically equivalent if $f-h$ is a sum of commutators in $\mathbb{F}\langle X\rangle$ and write $f \stackrel{\text { cyc }}{\sim} h$.

2.2. An effective Nullstellensatz. Let us recall an effective version of Hilbert's Nullstellensatz, giving bounds on the polynomials needed in the Bézout identity. We present a variant that combines [Kol88, Jel05]. Define

$$
\begin{aligned}
& N\left(n, d_{1}, \ldots, d_{r}\right)= \begin{cases}d_{1} \cdots d_{r} & \text { if } r \leq n, \\
d_{1} \cdots d_{n-1} d_{r} & \text { if } r>n>1, \\
d_{1}+d_{r}-1 & \text { if } r>n=1,\end{cases} \\
& N^{\prime}\left(n, d_{1}, \ldots, d_{r}\right)= \begin{cases}N\left(d_{1}, \ldots, d_{r}\right) & \text { if } r \leq n, \\
N\left(d_{1}, \ldots, d_{r}\right) & \text { if } r>n \geq 1 \text { and } d_{r}>2, \\
2 N\left(d_{1}, \ldots, d_{r}\right)-1 & \text { if } r>n>1 \text { and } d_{r} \leq 2, \\
2 d_{1}-1 & \text { if } r>n=1 \text { and } d_{r} \leq 2,\end{cases}
\end{aligned}
$$

for $d_{1} \geq \cdots \geq d_{r}$.

Theorem 2.1 (Kollár-Jelonek). Let $\mathbb{F}$ be an algebraically closed field and let $f_{1}, \ldots, f_{r} \in$ $\mathbb{F}\left[x_{1}, \ldots, x_{n}\right]$ be commutative polynomials without a common zero. Let $d_{i}=\operatorname{deg} f_{i}$ and assume $d_{1} \geq \cdots \geq d_{r}$. Then there exist $h_{1}, \ldots, h_{r} \in \mathbb{F}\left[x_{1}, \ldots, x_{n}\right]$ satisfying

$$
1=h_{1} f_{1}+\cdots+h_{r} f_{r}
$$

with $\operatorname{deg} h_{i} f_{i} \leq N^{\prime}\left(n, d_{1}, \ldots, d_{r}\right)$ for $1 \leq i \leq r$.

A core feature of this theorem we shall use is that the obtained degree bounds are independent of the number of variables $n$ (for large enough $n$ ).

2.3. Trace identities. An algebra with trace is an algebra $A$ equipped with an additional structure, that is a linear map $\operatorname{tr}: A \rightarrow A$ satisfying the following properties

$$
\operatorname{tr}(a b)=\operatorname{tr}(b a), \quad a \operatorname{tr}(b)=\operatorname{tr}(b) a, \quad \operatorname{tr}(\operatorname{tr}(a) b)=\operatorname{tr}(a) \operatorname{tr}(b)
$$

for all $a, b \in A$. A morphism between algebras with trace preserves the trace and such algebras form a category.

The free algebra in this category is the algebra of free noncommutative polynomials in the variables $x_{1}, \ldots, x_{g}$ over the polynomial algebra $T$ in the infinitely many variables $\operatorname{tr}(w)$, where $w$ runs over all representatives of the cyclic equivalence classes of words in the variables $x_{1}, \ldots, x_{g}$. Its elements are trace polynomials and elements of $T$ are pure trace polynomials. The degree of a trace monomial $\operatorname{tr}\left(w_{1}\right) \cdots \operatorname{tr}\left(w_{m}\right) v, w_{i}, v \in\langle X\rangle$, equals $|v|+\sum_{i}\left|w_{i}\right|$, where $|u|$ denotes the length of a word $u$. The degree of a trace polynomial is the maximum of the degrees of its trace monomials. 
Trace identities of the matrix algebra $M_{n}(\mathbb{F})$ are the elements in the kernel of the evaluation map from the free algebra with trace to $M_{n}(\mathbb{F})$. Pure trace identities are trace identities that belong to $T$. By [Pro76, Theorem 4.5], there are no trace identities of $M_{n}(\mathbb{F})$ of degree less than $n$ and no pure trace identities of degree less than $n+1$.

\section{Main Result}

Throughout this section let $\mathbb{F}$ be an algebraically closed field of characteristic 0 . The following is the main result of this paper. It is proved in Subsection 3.1 below. We remark that a very special case of Theorem 3.1 was obtained in [BK11] by different means.

Theorem 3.1 (Spurnullstellensatz). Let $f_{1}, \ldots, f_{r}, f \in \mathbb{F}\langle X\rangle$. The implication

$$
\operatorname{tr}\left(f_{1}(A)\right)=\cdots=\operatorname{tr}\left(f_{r}(A)\right)=0 \quad \Longrightarrow \quad \operatorname{tr}(f(A))=0
$$

holds for every $n$ and all $A \in M_{n}(\mathbb{F})^{g}$ if and only if $f$ is cyclically equivalent to a linear combination of $f_{i}$ 's or a linear combination of $f_{i}$ 's is cyclically equivalent to a nonzero scalar.

Remark 3.2. Note $\sum \lambda_{i} f_{i} \stackrel{\text { cyc }}{\sim} 1$ for some $\lambda_{i} \in \mathbb{F}$ does not necessarily imply that $f \stackrel{\text { cyc }}{\sim} \sum \mu_{i} f_{i}$ for some $\mu_{i} \in \mathbb{F}$. For example, take $f_{1}=1, f=x_{1}$.

Example 3.3. Theorem 3.1 fails in the dimension-dependent context: For each $n \in \mathbb{N}$ we give an example of polynomials $f_{1}, f_{2}$ such that $\operatorname{tr}\left(f_{1}\right), \operatorname{tr}\left(f_{2}\right)$ do not have a common zero on $M_{n}(\mathbb{F})$, but for which there do not exist $\lambda_{1}, \lambda_{2} \in \mathbb{F}$ and a polynomial identity $p$ of $M_{n}(\mathbb{F})$ such that

$$
\lambda_{1} f_{1}+\lambda_{2} f_{2} \stackrel{\text { cyc }}{\sim} 1+p .
$$

Let $c$ be a homogeneous central polynomial of $M_{n}(\mathbb{F})$, which means that $c(A) \in \mathbb{F}$ for all $A \in M_{n}(\mathbb{F})^{g}$ and $c$ does not vanish identically on $M_{n}(\mathbb{F})$ (see e.g. [Row80]). Take

$$
f_{1}=c, f_{2}=1+c^{2} .
$$

Then $\operatorname{tr}\left(f_{1}\right), \operatorname{tr}\left(f_{2}\right)$ do not have a common zero on $M_{n}(\mathbb{F})$. If (2) holds for some $\lambda_{1}, \lambda_{2} \in \mathbb{F}$ and a polynomial identity $p$ of $M_{n}(\mathbb{F})$, then

$$
\lambda_{1} \operatorname{tr}(c)+\lambda_{2}\left(\frac{1}{n} \operatorname{tr}(c)^{2}+n\right)=n .
$$

As $c$ is homogeneous, say of degree $k$, then

$$
\lambda_{1} \alpha^{k} \operatorname{tr}(c)+\lambda_{2}\left(\frac{1}{n} \alpha^{2 k} \operatorname{tr}(c)^{2}+n\right)=n
$$

for every $\alpha \in \mathbb{F}$. But $\mathbb{F}$ is infinite, so (3) cannot hold for every $\alpha \in \mathbb{F}$.

3.1. Proof of Theorem 3.1. As a first step towards the proof of Theorem 3.1 we prove its weaker variant, characterizing sets of polynomials $f_{i}$ whose traces do not have a common vanishing point.

Lemma 3.4. Let $f_{1}, \ldots, f_{r} \in \mathbb{F}\langle X\rangle$. If the equations

$$
\operatorname{tr}\left(f_{i}\left(x_{1}, \ldots, x_{g}\right)\right)=0,1 \leq i \leq r,
$$

do not have a common solution in $M(\mathbb{F})^{g}$, then $\sum \lambda_{i} f_{i} \stackrel{\text { cyc }}{\sim} 1$ for some $\lambda_{i} \in \mathbb{F}$. 
Proof. We can assume that $\operatorname{tr}\left(f_{i}\right)$ 's are linearly independent as elements in the free algebra with trace and we also assume that $\operatorname{tr}\left(f_{i}\right)$ cannot be written as $\operatorname{tr}\left(f_{i}^{\prime}\right)$ for a polynomial $f_{i}^{\prime}$ with $\operatorname{deg}\left(f_{i}^{\prime}\right)<\operatorname{deg}\left(f_{i}\right)$.

For every fixed $n$ we can evaluate a noncommutative polynomial $f$ on the symbolic $n \times n$ matrices $\xi_{k}=\left(x_{i j}^{(k)}\right), 1 \leq k \leq g$, and the trace of $f\left(\xi_{1}, \ldots, \xi_{g}\right)$ is a commutative polynomial in the variables $x_{i j}^{(k)}, 1 \leq i, j \leq n, 1 \leq k \leq g$. Note that $\operatorname{deg}(\operatorname{tr}(f)) \leq \operatorname{deg}(f)$ for every $f \in \mathbb{F}\langle X\rangle$, where $\operatorname{deg}(\operatorname{tr}(f))$ denotes the degree of the commutative polynomial $\operatorname{tr}\left(f\left(\xi_{1}, \ldots, \xi_{g}\right)\right)$. By Theorem 2.1, the condition (4) implies that there exist $h_{1}^{(n)}, \ldots, h_{r}^{(n)} \in \mathbb{F}\left[x_{i j}^{(k)}: 1 \leq i, j \leq\right.$ $n, 1 \leq k \leq g]$ such that

$$
1=\sum h_{i}^{(n)} \operatorname{tr}\left(f_{i}\right)
$$

with

$$
\operatorname{deg}\left(\operatorname{tr}\left(f_{i}\right)\right) \operatorname{deg}\left(h_{i}^{(n)}\right) \leq N^{\prime}\left(d_{1}, \ldots, d_{r}, n^{2} g\right),
$$

where $d_{i}=\operatorname{deg}\left(f_{i}\right)$ and $d_{1} \geq \cdots \geq d_{r}$. Applying the Reynolds operator (for the usual action of $\mathrm{GL}_{\mathrm{n}}$ on the polynomial ring $\mathbb{F}\left[x_{i j}^{(k)}: 1 \leq i, j \leq n, 1 \leq k \leq g\right]$; i.e., $\sigma \in \mathrm{GL}_{n}$ sends a variable $x_{i j}^{(k)}$ into the $(i, j)$-entry of the matrix $\left.\sigma^{-1}\left(x_{i j}^{(k)}\right) \sigma\right)$ to the equality (5) we can assume that the $h_{i}^{(n)}$ are invariant, and thus pure trace polynomials by [Pro76, Theorem 1.3]. The above bound (6) is independent of $n$ for $r \leq n^{2} g$ (see Theorem 2.1). Thus, the degree of $h_{i}^{(n)} \operatorname{tr}\left(f_{i}\right)$ can be in this case bounded above by $d_{1} \cdots d_{r}$.

We thus get for every sufficiently large $n$; i.e, for $n \geq d_{1} \cdots d_{r}$ and $n \geq \sqrt{\frac{r}{g}}$, a trace identity for $M_{n}(\mathbb{F})$,

$$
1=\sum h_{i}^{(n)} \operatorname{tr}\left(f_{i}\right)
$$

where $\operatorname{deg}\left(h_{i}^{(n)} \operatorname{tr}\left(f_{i}\right)\right) \leq n$. Let us fix $n$ with these properties. Since any nontrivial pure trace identity on $n \times n$ matrices has degree at least $n+1$ by [Pro76, Theorem 4.5], the above identity (7) must be trivial, which means that it holds in the free algebra with trace. A little care is needed at this point. If $f_{1}, \ldots, f_{r}$ do not all have zero constant term, then (7) is an identity in the free algebra with trace if we replace $\operatorname{tr}\left(f_{i}\right)$ by

$$
\tau_{i}=\operatorname{tr}\left(\overline{f_{i}}\right)+\alpha_{i} n, \quad 1 \leq i \leq r,
$$

where $\overline{f_{i}}$ is the sum of all nonconstant terms of $f_{i}$, and $\alpha_{i}$ is its constant term, and thus obtain an identity

$$
1=\sum h_{i}^{(n)} \tau_{i}
$$

Since all polynomials that appear in (8) are pure trace polynomials, they belong to the commutative subalgebra $T$ of the free algebra with trace, which is generated by the trace monomials $\operatorname{tr}\left(x_{i_{1}} \cdots x_{i_{t}}\right)$ modulo the relations $\operatorname{tr}\left(x_{i_{1}} x_{i_{2}} \cdots x_{i_{t}}\right)=\operatorname{tr}\left(x_{i_{2}} \cdots x_{i_{t}} x_{i_{1}}\right)$. Let $w_{1}, w_{2}, \ldots$ be the representatives of the cyclic equivalence classes of words in the variables $x_{1}, \ldots, x_{g}$. Then $T$ is the free commutative algebra generated by $t_{1}, t_{2}, \ldots$, where $t_{i}=\operatorname{tr}\left(w_{i}\right)$. 
Let us denote by $t_{0}$ the empty word; i.e., the identity of $T$, and write

$$
\tau_{i}=\sum_{j=1}^{m} \alpha_{i j} t_{j}+\alpha_{i m+1} t_{0} .
$$

Note that $\alpha_{i m+1}=\alpha_{i} n$. As we assumed that $\operatorname{tr}\left(f_{i}\right)$ are linearly independent, also $\tau_{i}$ are linearly independent. Indeed, assume that $\sum \lambda_{i} \tau_{i}=0$ for some $\lambda_{i} \in \mathbb{F}$. Then $\sum \lambda_{i} \operatorname{tr}\left(f_{i}\right)=0$ on $M_{n}(\mathbb{F})$ for the chosen $n \geq \operatorname{deg}\left(f_{i}\right)$. Thus, $\sum \lambda_{i} f_{i}$ has zero trace on $M_{n}(\mathbb{F})$, so it is cyclically equivalent to a polynomial identity on $M_{n}(\mathbb{F})$ [BK09, Theorem 4.5]. As $n \geq \operatorname{deg}\left(f_{i}\right)$ we can conclude that $\sum \lambda_{i} \operatorname{tr}\left(f_{i}\right)=0$ is an identity in the free algebra with trace and due to the assumption, $\lambda_{i}=0$ for all $i$.

Note that this implies that the matrix $\left(\alpha_{i j}\right)$ has linearly independent rows and can be brought to the reduced row echelon form. In particular, we can express $t_{i_{1}}, \ldots, t_{i_{r}}$ as linear combinations of $\tau_{1}, \ldots, \tau_{r}$ and $t_{i_{r+1}}, \ldots, t_{i_{m}}$, for some $\left\{i_{0}, i_{1}, \ldots, i_{m}\right\}=\{0,1, \ldots, m\}$. If the last row in the reduced echelon form of this matrix has all zeros except for the last entry, then $1=\sum \lambda_{i} \tau_{i}$. In this case $\operatorname{tr}\left(\sum \lambda_{i} f_{i}-\frac{1}{n}\right)=0$ on $M_{n}(\mathbb{F})$, which implies that $\sum \lambda_{i} f_{i}$ is cyclically equivalent to a nonzero scalar. Otherwise we can choose (free) generators of $T$ that include $\tau_{1}, \ldots, \tau_{r}$ and the above identity (8) cannot hold since it does not hold in the free commutative algebra.

Proof of Theorem 3.1. To prove the nontrivial direction, assume that no linear combination of $f_{i}$ 's is cyclically equivalent to a nonzero scalar. The condition (1) implies that the equations

$$
\operatorname{tr}\left(f_{i}\left(x_{1}, \ldots, x_{g}\right)\right)=0 \quad(1 \leq i \leq r), \quad \operatorname{tr}\left(f\left(x_{1}, \ldots, x_{g}\right)+1\right)=0
$$

do not have a common solution in $M(\mathbb{F})$. Hence

$$
\sum \lambda_{i} f_{i}+\lambda(1+f) \stackrel{\text { cyc }}{\sim} 1
$$

for some $\lambda_{i}, \lambda \in \mathbb{F}$ by Lemma 3.4. By our assumption, $\lambda \neq 0$. Thus,

$$
f \stackrel{\text { cyc }}{\sim} \sum \mu_{i} f_{i}+\mu
$$

for some $\mu_{i}, \mu \in \mathbb{F}$. If $\mu \neq 0$, then the initial condition (1) is violated again by our assumption and Lemma 3.4. Hence $\mu=0$ and $f \stackrel{\text { cyc }}{\sim} \sum \mu_{i} f_{i}$.

3.2. Bounds on the size of matrices in Theorem 3.1. The proof of Lemma 3.4 reveals a bound on the size of matrices for which it suffices to test the condition of this lemma and of Theorem 3.1 in order to draw the conclusion. If the implication (1) holds for all $A \in M_{N}(\mathbb{F})^{g}$ for $N=\max \left\{d_{1} \cdots d_{r} d, \sqrt{\frac{g}{r}}\right\}$, where $d_{i}=\operatorname{deg} f_{i}, d=\operatorname{deg} f$, then it holds for all $A \in M_{n}(\mathbb{F})^{g}$ for all $n \in \mathbb{N}$. In view of Theorem 2.1 we can sometimes sharpen this bound in the case that there exists $m$ satisfying $d_{1} \cdots d_{m^{2} g} d_{r} \leq m<\sqrt{\frac{g}{r}}$.

\subsection{Passing between a real closed field and its algebraic closure.}

Proposition 3.5. Let $R$ be a real closed field (e.g. $R=\mathbb{R})$ and let $C$ be its algebraic closure. For polynomials $f_{1}, \ldots, f_{r}, f \in R\langle X\rangle$ the following conditions are equivalent:

(i) For every $n \in \mathbb{N}$ and all $A \in M_{n}(C)^{g}$ we have $\operatorname{tr}\left(f_{1}(A)\right)=\cdots=\operatorname{tr}\left(f_{r}(A)\right)=0$ implies $\operatorname{tr}(f(A))=0$; 
(ii) There exist $\lambda_{i} \in C$ such that $\sum \lambda_{i} f_{i} \stackrel{\text { cyc }}{\sim} 1$ or $\sum \lambda_{i} f_{i} \stackrel{\text { cyc }}{\sim} f$;

(iii) There exist $\lambda_{i} \in R$ such that $\sum \lambda_{i} f_{i} \stackrel{\text { cyc }}{\sim} 1$ or $\sum \lambda_{i} f_{i} \stackrel{\text { cyc }}{\sim} f$;

(iv) For every $n \in \mathbb{N}$ and all $A \in M_{n}(R)^{g}$ we have $\operatorname{tr}\left(f_{1}(A)\right)=\cdots=\operatorname{tr}\left(f_{r}(A)\right)=0$ implies $\operatorname{tr}(f(A))=0$.

Proof. By Theorem 3.1, (i) is equivalent to (ii). By taking real parts, it is easy to see that (ii) implies (iii). The implication (iii) to (iv) is trivial. We will prove that (ii) follows by assuming (iv). To obtain a contradiction suppose first that the equations

$$
\operatorname{tr}\left(f_{i}\left(x_{1}, \ldots, x_{g}\right)\right)=0,1 \leq i \leq r
$$

do not have a common solution in $M(R)$ but do have one in $M(C)$. Let $a_{1}, \ldots, a_{g} \in M_{n}(C)$ be such that $\operatorname{tr}\left(f_{i}\left(a_{1}, \ldots, a_{g}\right)\right)=0$ for $1 \leq i \leq r$. Write $a_{j}=b_{j}+\dot{\mathrm{i}} c_{j}$, where $b_{j}, c_{j} \in M_{n}(R)$, and define

We have

$$
\widetilde{a_{j}}=\left(\begin{array}{cc}
b_{j} & c_{j} \\
-c_{j} & b_{j}
\end{array}\right)
$$

$$
\operatorname{tr}\left(f_{i}\left(\widetilde{a_{1}}, \ldots, \widetilde{a_{g}}\right)\right)=2 \operatorname{Re}\left(\operatorname{tr}\left(f_{i}\left(a_{1}, \ldots, a_{g}\right)\right)\right)=0
$$

for every $1 \leq i \leq r$. Thus, $\widetilde{a_{1}}, \ldots, \widetilde{a_{g}}$ is a common solution of the equations $(9)$ in $M(R)$, a contradiction. By Lemma 3.4 we therefore have $\sum \lambda_{i} f_{i} \stackrel{\text { cyc }}{\sim} 1$ for some $\lambda_{i} \in C$.

If the system (9) does have a solution in $M(R)$, then (iv) implies that the equations

$$
\operatorname{tr}\left(f_{i}\left(x_{1}, \ldots, x_{g}\right)\right)=0, \operatorname{tr}\left(1+f\left(x_{1}, \ldots, x_{g}\right)\right)=0
$$

do not have a common solution in $M(R)$, and by the previous step, applied to polynomials $f_{1}, \ldots, f_{r}, 1+f$, they also do not have a solution in $M(C)$. By Lemma $3.4, f \stackrel{\text { cyc }}{\sim} \sum \lambda_{i} f_{i}+\lambda 1$ for some $\lambda_{i}, \lambda \in C$. Since we are assuming that the equations (9) have a common solution in $M(R)$, (iv) implies $\lambda=0$.

\section{A tracial moment problem}

The main result of this section, Corollary 4.2, solves a constrained truncated tracial moment problem. For its proof we dualize the statement of Theorem 3.1. We refer the reader to [Bur11] and the references therein for more details on tracial moment problems.

Let $\mathbb{F} \in\{\mathbb{R}, \mathbb{C}\}$. We say that a linear functional $L: \mathbb{F}\langle X\rangle_{d} \rightarrow \mathbb{F}$ is tracial if it vanishes on sums of commutators, or equivalently, if $L(v)=L(w)$ for $v \stackrel{\text { cyc }}{\sim} w$. The simplest examples of such $L$ are obtained as follows. For $A \in M_{n}(\mathbb{F})^{g}$ define

$$
\phi_{A}: \mathbb{F}\langle X\rangle_{d} \rightarrow \mathbb{F}, \quad \phi_{A}(p)=\operatorname{tr}(p(A)),
$$

and let

$$
\mathcal{C}=\operatorname{span}\left\{\phi_{A} \mid A \in M_{n}(\mathbb{F})^{g}, n \in \mathbb{N}\right\} \subseteq \mathbb{F}\langle X\rangle_{d}^{*} .
$$

Tracial linear functionals can be described in terms of moment sequences. A sequence $\left(\alpha_{w}\right)_{w \in\langle X\rangle_{d}}$ in $\mathbb{F}$ is a truncated tracial moment sequence if $\alpha_{w}=\alpha_{v}$ for $v \stackrel{\text { cyc }}{\sim} w$. Note that any element in $\mathcal{C}$ is tracial and defines a (truncated) tracial moment sequence.

Proposition 4.1. If $L$ is a tracial linear functional on $\mathbb{F}\langle X\rangle_{d}$, then $L \in \mathcal{C}$. 
Proof. If $L \notin \mathcal{C}$, then there exists a linear functional $p \in\left(\mathbb{F}\langle X\rangle_{d}^{*}\right)^{*} \cong \mathbb{F}\langle X\rangle_{d}$ such that $p(\phi)=0$ for every $\phi \in \mathcal{C}$ and $p(L)=1$. By definition of $\mathcal{C}$ we have $\operatorname{tr}(p(A))=0$ for all $A \in M_{n}(\mathbb{F})^{g}$, $n \in \mathbb{N}$, which implies $p \stackrel{\text { cyc }}{\sim} 0$ (see e.g. [Pro76, Corollary 4.4]). As $L$ is tracial, $p(L)=L(p)=0$, a contradiction.

To give an explicit representation for the tracial linear functional $L$ as a linear combination of the $\phi_{A}$ with $g$-tuples $A$ of $d \times d$ matrices, we present an alternative constructive proof of Proposition 4.1.

Alternative proof. Given a tracial moment sequence $(L(w))_{w \in\langle X\rangle_{d}}$ we show how to find $g$ tuples $A_{\ell}$ such that $L(p)=\sum \lambda_{\ell} \phi_{A_{\ell}}$. We proceed inductively. Assume that there exist $A_{\ell}$, $1 \leq \ell \leq \ell_{m}$, such that $L(p)=\sum \phi_{A_{\ell}}$ for all $p \in \mathbb{F}\langle X\rangle_{m}^{\prime}$. We want to find an element of $\mathcal{C}$ which coincides with $L$ on $\mathbb{F}\langle X\rangle_{m+1}^{\prime}$. Define $L_{m+1}=L-\sum_{\ell=1}^{\ell_{m}} \phi_{A_{\ell}}$. It is enough to choose matrices $a_{\ell 1}, \ldots, a_{\ell g} \in M_{m+1}(\mathbb{F}), \ell_{m}+1 \leq \ell \leq \ell_{m+1}$, such that $\operatorname{tr}\left(p\left(a_{\ell 1}, \ldots, a_{\ell g}\right)\right)=0$ for $p \in \mathbb{F}\langle X\rangle_{m}^{\prime}$ and $L_{m+1}(p)=\sum_{\ell=\ell_{m}+1}^{\ell_{m+1}} \operatorname{tr}\left(p\left(a_{\ell 1}, \ldots, a_{\ell g}\right)\right)$ for $p$ homogeneous of degree $m+1$.

Choose representatives $w_{\ell_{m}+1}, \ldots, w_{\ell_{m+1}}$ of cyclic equivalence classes of words in the variables $x_{1}, \ldots, x_{g}$ of degree $m+1$. Let $w_{\ell}=x_{i_{1}}^{j_{1}} \cdots x_{i_{s}}^{j_{s}}$, where $\sum_{k} j_{k}=m+1$. We denote $s_{k}=\sum_{i=1}^{k} j_{i}$. Setting $a_{\ell i}=0$ at the beginning, we define matrices $a_{\ell i} \in M_{m+1}(\mathbb{F})(1 \leq i \leq g)$ as follows. We let $k$ vary from 1 to $s$, and at step $k$ we replace $a_{\ell i_{k}}$ by

$$
\begin{cases}a_{\ell i_{k}}+\sum_{u=s_{k-1}+1}^{s_{k}} e_{u, u+1} & \text { if } k<s, \\ a_{\ell i_{k}}+\sum_{u=s_{k-1}+1}^{s_{k}-1} e_{u, u+1}+L_{m+1}\left(w_{\ell}\right) e_{m+1,1} & \text { if } k=s .\end{cases}
$$

Here $e_{i j}$ are the standard $(m+1) \times(m+1)$ matrix units.

We claim that the only word in $a_{\ell 1}, \ldots, a_{\ell g}$ of degree $\leq m+1$ with nonzero trace is cyclically equivalent to $w_{\ell}$. A necessary condition for a word $w$ in $a_{\ell 1}, \ldots, a_{\ell g}, w=a_{\ell p_{1}}^{r_{1}} \cdots a_{\ell p_{s}}^{r_{s}}$, of degree $m^{\prime}, 1 \leq m^{\prime} \leq m+1$, to have nonzero trace is the existence of a sequence $\left(\tilde{e}_{j}\right)_{j=1}^{m+1}$ of matrix units from the set $E=\left\{e_{u, u+1}, e_{m+1,1} \mid 1 \leq u \leq m\right\}$ such that $\operatorname{tr}\left(\tilde{e}_{1} \cdots \tilde{e}_{m+1}\right)=1$, and if $\sum_{i=1}^{k-1} r_{i}<j \leq \sum_{i=1}^{k} r_{i}$ then $\tilde{e}_{j}$ appears in $a_{\ell p_{k}}$. The product of the elements in $E$ has nonzero trace only in a unique order (up to cyclic permutations). Since every element in $E$ appears only in one $a_{\ell i}$, this order determines $p_{1}, \ldots, p_{s}$. Thus, $\operatorname{tr}\left(w\left(a_{\ell 1}, \ldots, a_{\ell g}\right)\right)=L_{m+1}(w)$ for $w \stackrel{\text { cyc }}{\sim} w_{\ell}$ and 0 otherwise. Therefore, $a_{\ell 1}, \ldots, a_{\ell g}, \ell_{m}+1 \leq \ell \leq \ell_{m+1}$, have the desired properties.

We have thus found $g$-tuples $A_{\ell} \in M_{n_{\ell}}(\mathbb{F})^{g}, 1 \leq \ell \leq \ell_{d}$, such that $L(p)=\sum \phi_{A_{\ell}}(p)$ for every $p \in \mathbb{F}\langle X\rangle_{d}^{\prime}$. Take $A_{0}=(0, \ldots, 0) \in \mathbb{F}^{g}$, and notice that $L(p)=\sum \phi_{A_{\ell}}(p)+(L(\varnothing)-n) \phi_{A_{0}}(p)$, where $n=\sum \phi_{A_{\ell}}(1)=\sum n_{\ell}$, for every $p \in \mathbb{F}\langle X\rangle_{d}$.

Let us fix polynomials $f_{1}, \ldots, f_{r} \in \mathbb{F}\langle X\rangle_{d}$ and write $f_{i}=\sum \lambda_{i j} w_{j}$. We say that a sequence $(L(w))_{w \in\langle X\rangle_{d}}$ is a constrained truncated tracial moment sequence if it is a truncated tracial moment sequence and if $L\left(f_{i}\right)=\sum_{j} \lambda_{i j} L\left(w_{j}\right)=0$ for $1 \leq i \leq r$. We define a constrained analog of $\mathcal{C}$,

$$
\mathcal{S}=\operatorname{span}\left\{\phi_{A} \in \mathcal{C} \mid \phi_{A}\left(f_{i}\right)=0,1 \leq i \leq r\right\}
$$

Note that every element of $\mathcal{S}$ defines a constrained truncated tracial moment sequence. 
Corollary 4.2 (Constrained truncated tracial moment problem). If $(L(w))_{w \in\langle X\rangle_{d}}$ is a constrained tracial moment sequence with $L(1)=1$, then $L \in \mathcal{S}$.

Proof. If $L \notin \mathcal{S}$ then there exists an element $p \in\left(\mathbb{F}\langle X\rangle_{d}^{*}\right)^{*}$ such that $p(\phi)=0$ for all $\phi \in \mathcal{S}$ and $p(L)=1$. We have $\operatorname{tr}(p(A))=0$ for all $A \in M_{n}(\mathbb{F})^{g}$ with the property $\operatorname{tr}\left(f_{i}(A)\right)=0$ for all

$1 \leq i \leq r$. Thus, Theorem 3.1 and Proposition 3.5 imply that $p \stackrel{\text { cyc }}{\sim} \sum \lambda_{i} f_{i}$ or $\sum \lambda_{i} f_{i} \stackrel{\text { cyc }}{\sim} 1$ for some $\lambda_{i} \in \mathbb{F}$. In the former case we have $L(p)=\sum \lambda_{i} L\left(f_{i}\right)=0$, which contradicts $L(p)=1$, in the last case $L(1)=\sum \lambda_{i} L\left(f_{i}\right)=0$, which is contrary to the assumption $L(1)=1$.

Acknowledgments. This paper was written while the second author was visiting The University of Auckland. She would like to thank the Department of Mathematics for its hospitality.

\section{REFERENCES}

[Ami57] S. A. Amitsur, A generalization of Hilbert's Nullstellensatz, Proc. Amer. Math. Soc. 8 (1957), 649656. 1

[BK09] M. Brešar, I. Klep, Noncommutative Polynomials, Lie Skew-Ideals and Tracial Nullstellensätze, Math. Res. Lett. 16 (2009), 605-626. 5

[BK11] M. Brešar, I. Klep, Tracial Nullstellensätze, in: "Notions of Positivity and the Geometry of Polynomials" (J. Borcea memorial volume) edited by P. Brändén, M. Passare and M. Putinar, 79-101, Birkhäuser, 2011. 1, 3

[Bur11] S. Burgdorf, Trace-positive polynomials, sums of hermitian squares and the tracial moment problem, Ph.D. Thesis, Universität Konstanz and Université de Rennes 1, 2011. 6

[CHMN13] J. Cimprič, J. W. Helton, S. McCullough, C. Nelson, A noncommutative real Nullstellensatz corresponds to a noncommutative real ideal: Algorithms, Proc. Lond. Math. Soc. (3) 106 (2013), 1060-1086. 1

[CGM09] B. Collins, A. Guionnet, E. Maurel-Segala, Asymptotics of unitary and orthogonal matrix integrals, Adv. Math. 222 (2009), 172-215. 1

[HM04] J. W. Helton, S. McCullough, A Positivstellensatz for non-commutative polynomials, Trans. Amer. Math. Soc. 356 (2004), 3721-3737. 1

[Jel05] Z. Jelonek, On the effective Nullstellensatz, Invent. Math. 162 (2005), 1-17. 1, 2

[KS08] I. Klep, M. Schweighofer, Connes' embedding conjecture and sums of hermitian squares, Adv. Math. 217 (2008), 1816-1837. 1

[Kol88] J. Kollár, Sharp effective Nullstellensatz, J. Amer. Math. Soc. 1 (1988), 963-975. 1, 2

[Pro76] C. Procesi, The invariant theory of $n \times n$ matrices, Adv. Math. 19 (1976), 306-381. 1, 3, 4, 7

[Row80] L. H. Rowen, Polynomial identities in ring theory, Academic Press, 1980. 1, 3

[Som99] M. Sombra, A sparse effective Nullstellensatz, Adv. in Appl. Math. 22 (1999), 271-295. 1

Igor Klep, Department of Mathematics, The University of Auckland, New Zealand

E-mail address: igor.klep@auckland.ac.nz

Špela Špenko, Institute of Mathematics, Physics, and Mechanics, Luubluana, Slovenia

E-mail address: spela.spenko@imfm.si 